\title{
CD95 ligand (CD95L) immunohistochemistry: a critical study on 12 antibodies
}

\author{
J Sträter ${ }^{\star, 1}$, H Walczak², C Hasel ${ }^{1}$, I Melzner ${ }^{1}$, \\ F Leithäuser ${ }^{1}$ and P Möller ${ }^{1}$ \\ 1 Institute of Pathology, University of Ulm, Albert-Einstein-Allee 11, D-89081 \\ Ulm, Germany \\ 2 Apoptosis Research Group (G0310), Tumor Immunology Program, Deutsches \\ Krebsforschungszentrum, Im Neuenheimer Feld 280, D-69120 Heidelberg, \\ Germany \\ * Corresponding author: Dr. J Sträter, Institute of Pathology, University of UIm, \\ Albert-Einstein-Allee 11, D-89081 Ulm, Germany. \\ Tel: +49-731-5002-3320; Fax: +49-731-5002-3884; \\ E-mail: joern.straeter@medizin.uni-ulm.de
}

Received 10.7.00; revised 7.11.00; accepted 15.11 .00

Edited by D Vaux

\begin{abstract}
In recent years, some studies on the expression of CD95(Fas/ APO-1) ligand (CD95L) in tissues or cells raised concerns about the specificity of the antibodies used. We therefore tested 12 CD95L antibodies for their reliability in immunocyto/ histochemistry by (i) staining CD95L-transfected and control CV-1/EBNA cells and (ii) comparing staining patterns in immunohistochemically labeled tissue sections with the localization of $\mathrm{CD} 5 \mathrm{~L}^{+}$cells in in situ hybridization. While G247-4, NOK-1, NOK-2, 4H9, and MIKE-1 stained CD95Ltransfected cells and did not significantly bind to controls, G247-4 was the only antibody giving satisfying signals in tissue sections perfectly matching the distribution of $\mathrm{CD} 95 \mathrm{~L}^{+}$ cells by in situ hybridization. MAb $33, \mathrm{C}-20$, and $\mathrm{N}-20$ comparably stained both transfected and control cells and showed considerable background or falsely positive staining in sections. MIKE-2, 8B8, A11, and 4A5 did not or only very faintly bind to either cells and, thus, were not tested on sections. We conclude that G247-4 is the only tested antibody that is recommendable for immunohistochemistry. Cell Death and Differentiation (2001) 8, 273-278.
\end{abstract}

Keywords: antibody; CD95 ligand; immunohistochemistry

Abbreviations: CD95L, CD95 ligand; FACS, fluorescenceassisted cell scan; kDa, kilo Dalton; mAb, monoclonal antibody; mRNA, message ribonucleic acid; PBS, phosphate buffered saline; RT-PCR, reverse transcriptase-polymerase chain reaction; TRAIL, tumor necrosis factor-related apoptosis-inducing ligand

\section{Introduction}

The CD95(Fas/APO-1) ligand (CD95L) is a $40 \mathrm{kDa}$ type II transmembrane protein and is a member of the tumor necrosis factor superfamily of cytokines., ${ }^{1,2}$ It induces apoptosis in CD95-expressing and -sensitive cells and has been implicated in a variety of conditions such as the regulation of inflammatory responses, ${ }^{3}$ maintenance of the immune privilege of certain tissues, ${ }^{4}$ and the pathogensis of cancer ('CD95L counterattack' hypothesis). ${ }^{5}$ Since its discovery in 1992, a variety of studies using different polyclonal and monoclonal antibodies for the detection of the protein in Western blot analysis and immunohistochemistry has been published. Some of these studies, however, raised serious concerns about the specificity of some broadly applied reagents. ${ }^{6-10}$ In the present study, we therefore critically tested 12 different commercially available CD95L antibodies for their reliability in immunohistochemistry. This was done (i) by staining CD95L-transfected and control CV-1/EBNA cells and (ii) by comparing staining patterns in immunohistochemistry on tissue sections with the distribution of CD95L mRNA expressing cells by in situ hybridization.

\section{Results \\ Immunocytochemistry on CD95L-transfected CV-1/EBNA cells}

To test the specificity of 12 different commercially available CD95L antibodies in immunocyto/histochemistry, immunocytochemical stainings were performed on CV-1/EBNA cells transfected with full length human CD95L. Cells transfected with human TRAIL and with vector alone served as controls. CD95L- and TRAIL- expression of transfected cells was controled by RT-PCR (Figure 1). Efficiency of CD95Ltransfection was also checked by in situ hybridization (data not shown).

The staining results with CD95L- and TRAIL-transfected cells are given in Figure 2. Briefly, only staining with G247-4, NOK-1, and NOK-2 gave clear positive staining results on CD95L-transfected cells while being negative in controls. MIKE-1 labeled CD95L-transfected cells but also showed slight background staining in few control cells. Staining of CD95L-transfected cells with $4 \mathrm{H} 9$ was very weak, but control cells were clearly negative. In contrast, both polyclonal antibodies tested (C-20 and $\mathrm{N}$ 20) as well as the monoclonal antibody 33 did not only stain CD95L-transfected cells but gave an almost identical staining result in control cells. In addition, staining with MIKE-2 gave a comparable, although very faint, staining of both CD95L-transfected and control cells. All other antibodies tested gave negative staining results on either cells.

Similar staining results as with TRAIL-transfected cells were obtained with CV-1/EBNA cells transfected with vector alone (data not shown). 


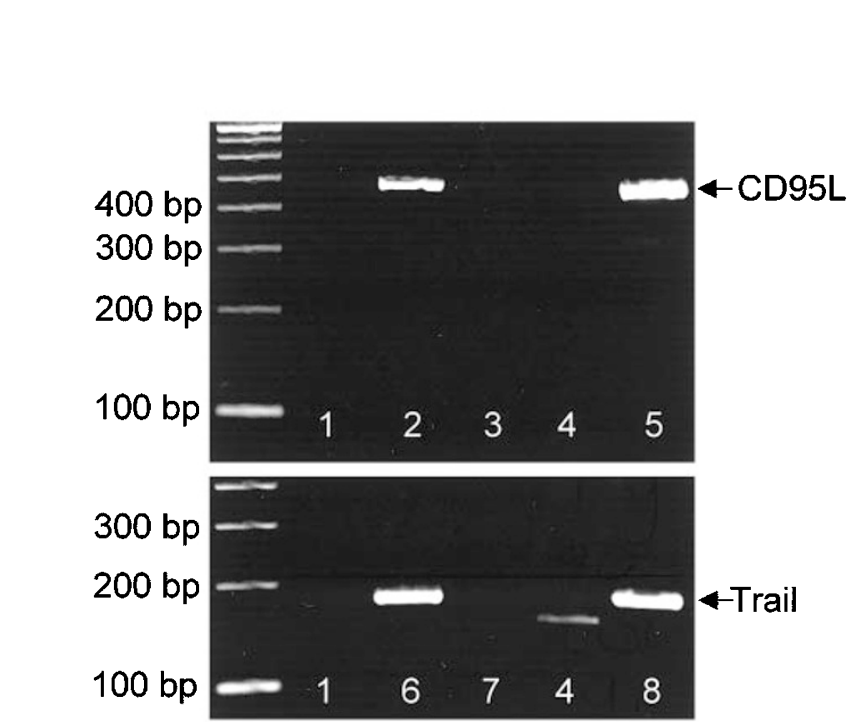

Figure 1 CD95L- and TRAIL-expression in transfected CV-1/EBNA cells as revealed by RT - PCR for CD95L (upper panel) and TRAIL (lower panel). Lane 1: cDNA of vector-transfected cells; lane 2: CDNA of CD95L-transfected cells; lane 3: RNA of CD95L-transfected cells; lane 4: DNA of vector-transfected cells; lane 5: DNA of CD95L-transfected cells; lane 6: CDNA of TRAILtransfected cells; lane 7: RNA of TRAIL-transfected cells; lane 8: DNA of TRAIL-transfected cells. The faint band in lane 4 in the lower panel is probably due to amplification of monkey TRAIL DNA

\section{CD95L immunohistochemistry on tonsillar tissue sections}

Using the antibodies which gave clear positive staining results on CD95L-transfected cells (irrespective of specificity), we next carried out immunohistochemical staining on normal tissue using both frozen and paraffin tonsillar sections. We found that after antibody-specific antigen retrieval (cf. Materials and Methods) the staining patterns in paraffin sections were very similar to those obtained in frozen sections although the reaction was somewhat weaker in paraffin compared to frozen sections.

To further evaluate the specificity of the antibodies used, we compared the immunohistochemical staining patterns to those obtained by in situ hybridization. CD95L-mRNApositive (immune) cells were detected mostly near the tonsillar crypt epithelium by in situ hybridization (Figure 3a). As already reported, ${ }^{11}$ plasma cells, recognized by their typical broad cytoplasmic rim with an excentrically located nucleus, were prominent producers of CD95L mRNA in the tonsil (Figure $3 a$, insert). In addition, some scattered lymphoid cells in the paracortical T-cell area and very few cells in the germinal centers expressed CD95L mRNA (not shown). Notably, the squamous epithelium was CD95L mRNA negative.

In immunohistochemistry, we revealed an almost identical staining pattern with the antibody G247-4 (Figure $3 b$ ). In contrast, the polyclonal antibodies C-20 (Figure 3c) and N-20 (Figure $3 \mathrm{~d}$ ) gave a rather diffuse staining of the squamous epithelium and immune cells. In some areas, plasma cells seemed to be stained somewhat more intensely than the adjacent epithelium, but this labeling

could not always be differentiated from the high background reaction. The monoclonal antibody 33 most strongly labeled the squamous epithelium (Figure $3 e$ ) as well as germinal center cells (not shown), but also a variety of immune cells in the interfollicular area, although less intensely.

NOK-1, NOK-2, MIKE-1, and 5H9 gave rise to a faint labeling of some lymphoid and plasma cells in frozen sections (not shown) but did not sufficiently stain CD95Lexpressing cells in paraffin sections (Figure 3f).

\section{Discussion}

Already some years ago, a publication by Giordano and co-workers $^{12}$ on CD95L-expression in thyrocytes provoked comments expressing serious concerns about the specificity of some of the commercially available CD95L antibodies in immunoblots, FACS analysis and immune fluorescence. ${ }^{6,7}$ The antibodies in question were the mouse monoclonal antibody 33 (IgG1 isotype; Transduction Laboratories, Lexington, KY, USA) and the rabbit polyclonal C-20 (purified IgG; Santa Cruz Biotechnology, Santa Cruz, CA, USA). Despite these published notes of caution, both antibodies, together with another rabbit polyclonal antibody, N-20 (Santa Cruz Biotechnology), have been widely used in studies for the in situ localization of CD95L in tissue sections by immunohistochemistry. The current flood of publications using antibodies which may lack sufficient specificity, however, requires a critical reevaluation of the reliability of available CD95L antibodies. We therefore tested the specificity of 12 different CD95L antibodies in immunocyto/histochemistry by staining of CD95L-transfected and (TRAIL- and vector-transfected) control cells. These stainings demonstrate that only NOK1, NOK-2, G247-4, MIKE-1, and 4H9 have satisfying specificity in immunocyto/histochemistry. Both polyclonal antibodies tested ( $\mathrm{C}-20$ and $\mathrm{N}-20$ ) as well as $\mathrm{mAb} 33$ label CD95L-transfected and control cells to a comparable extent and, thus, lack sufficient specificity. Specificity of the monoclonal CD95L antibody 33 has first been doubted when CD95L was found in normal thyrocytes in Western blots using $\mathrm{mAb} 33$ although they lacked detectable CD95L mRNA in both RT-PCR and nuclease protection assays. ${ }^{6}$ Fiedler et al. ${ }^{7,9}$ reported that, in immunoblots, $\mathrm{mAb} 33$ detected an identical $37 \mathrm{kDa}$ signal in both CD95Ltransfected and untransfected 293T cells while, using mAb G247-4, only transfected cells showed a CD95Lspecific signal. Moreover, mAb 33 did not recognize immunoprecipitated CD95L from transfected 293T cells, but a $37 \mathrm{kDa}$ protein in the supernatant of the immunoprecipitate. $^{7}$ Finally, in 2D electrophoresis, mAb 33 detected a protein with different mobility than the CD95L specific signal detected by G247-4 and a polyclonal rabbit anti-CD95L antibody, PE62. ${ }^{9}$ In our present study, mAb33 did not only stain CD95L-transfected and control cells to a similar extent, but also strongly labeled tonsillar epithelial cells in tissue sections which lacked CD95L mRNA by in situ hybridization. Even though there may be differences in binding properties under reducing and non-reducing conditions, ${ }^{9,13}$ our findings support the assumption by 


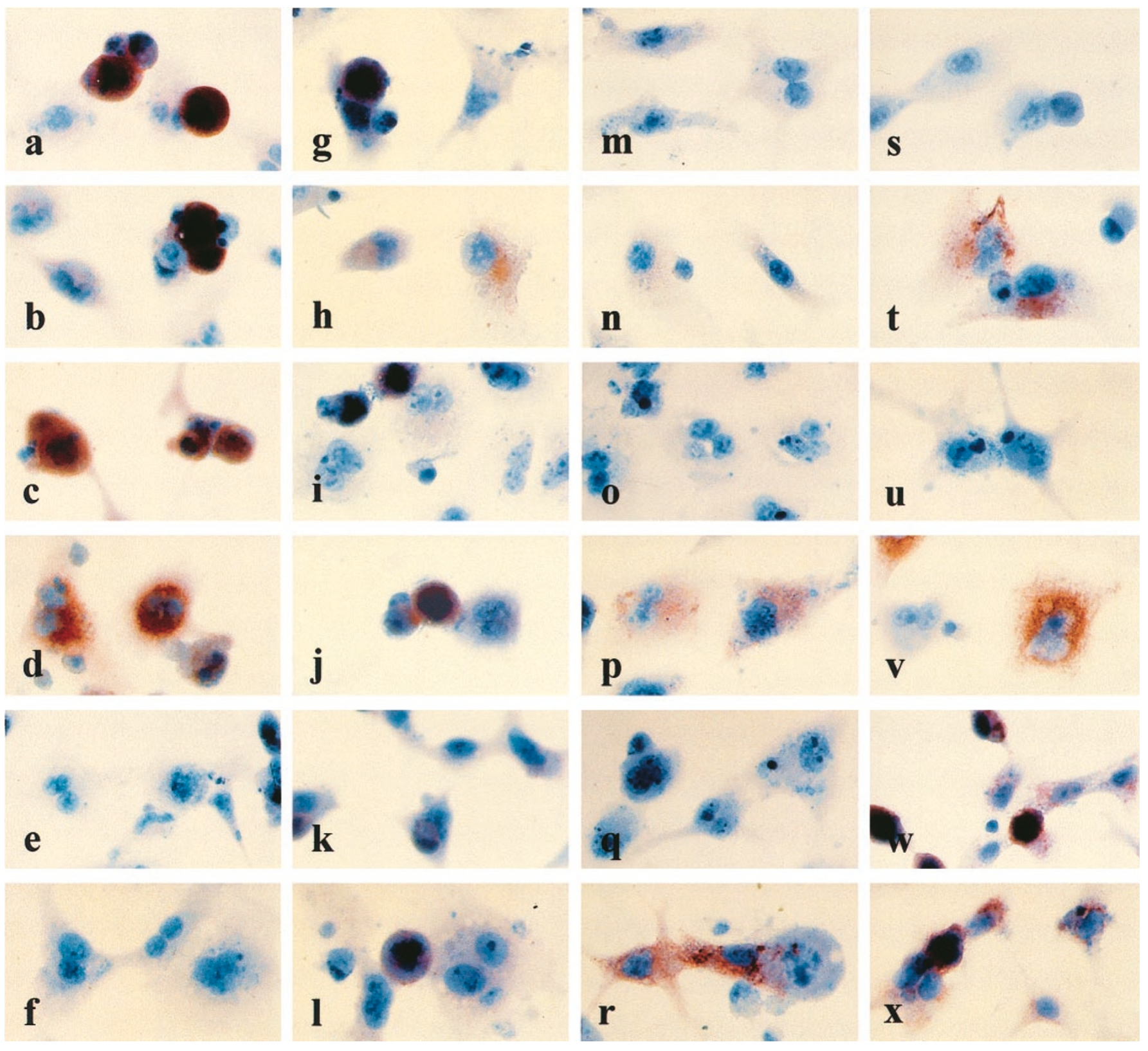

Figure 2 CD95L immunocytochemistry on CV-1/EBNA cells transfected with full length TRAIL (a, c, e, g, i, k, m, o, q, s, u, w) and CD95L (b, d, f, h, j, l, n, p, r, t, $\mathbf{v}, \mathbf{x})$, respectively. The antibodies used were C-20 (a, b), N-20 (c, d), A11 (e, f), MIKE-1 (g, h), MIKE-2 (i, j), 8B8 (k, I), 4A5 (m, n), 4H9 (o, p), G247-4 (q, r), NOK-1 $(\mathbf{s}, \mathbf{t})$, NOK-2 $(\mathbf{u}, \mathbf{v})$, and M33 $(\mathbf{w}, \mathbf{x})$. Original magnification $\times 300$

Fiedler and co-workers ${ }^{9}$ that $\mathrm{mAb} 33$ most probably detects a protein different from CD95L. In any case, it can not be recommended for use in immunohistochemistry.

Another antibody giving rise to debates on its specificity is the polyclonal rabbit anti-CD95L antibody C-20. Although C-20 recognizes CD95L in CD95L-transfected 293T cells in immunoblots and immunofluorescence, there is a strong background staining in control cells. ${ }^{7}$ In FACS analyses, C20 was shown to bind to unstimulated Jurkat cells which were, however, negative with the CD95L mAbs NOK-1 and Alf-1.2. ${ }^{8}$ These reports are in line with our immunohistochemical data showing that $\mathrm{C}-20$ stains both CD95L- transfected and control cells and gives rise to a considerable background reaction which is not clearly distinguishable from a possible additional CD95L-specific signal.

The very same applies to another polyclonal rabbit antiCD95L antibody supplied by Santa Cruz Biotechnology, N20. In our study, this antibody also stained CD95Ltransfected and control cells to a similar extent. In tissue sections, the labeling of plasma cells known to be the most prominent producers of CD95L in the tonsil and clearly positive with mAb G247-4 and by in situ hybridization, is not sufficiently distinct from the background reaction. In 

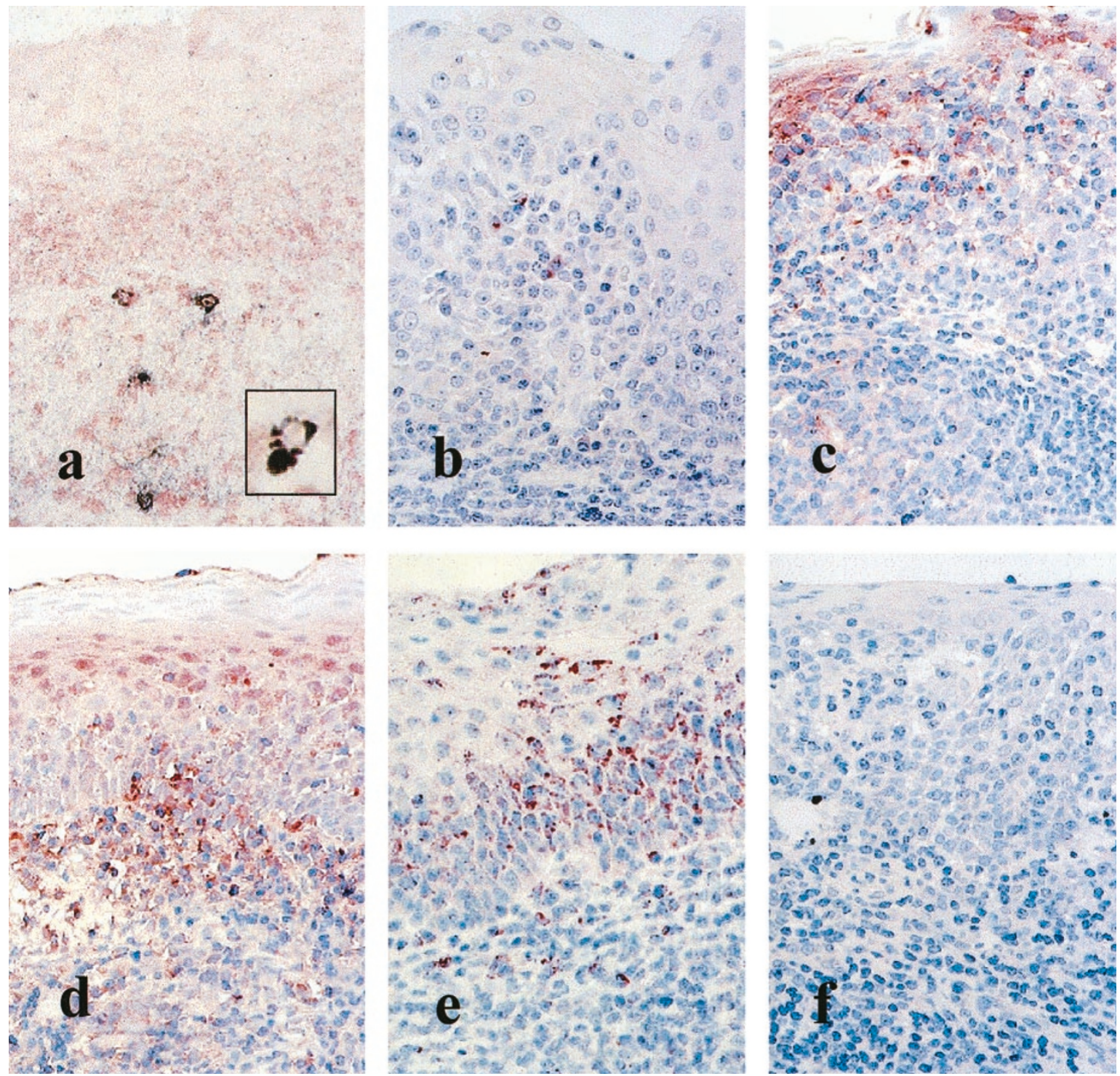

Figure 3 In situ hybridization of CD95L mRNA (a) and immunohistochemical stainings of tonsillar paraffin sections with Abs G247-4 (b), C-20 (c), N-20 (d), 33 (e), and NOK-1 (f) (original magnification $\times 120$ ). Insert in (a) highlights a typical plasma cell expressing CD95L mRNA (original magnification $\times 340$ )

conclusion, both polyclonal antibodies do not seem appropriate for use in immunohistochemistry.

Of all antibodies with sufficient specificity in immunocytochemistry on transfected cells (NOK-1, NOK-2, G247-4, MIKE-1, 4H9), only G247-4 showed a satisfying sensitivity in stainings of tonsillar tissue sections. The staining pattern obtained with this antibody on frozen as well as paraffin sections perfectly matched the distribution of CD95L mRNA-expressing cells by in situ hybridization. However, the sensitivity in paraffin sections was somewhat lower. NOK-1, NOK-2, MIKE-1, and $4 \mathrm{H} 9$ faintly stained only very few lymphoid and plasma cells in frozen sections and did not have satisfying sensitivity in paraffin sections. Thus, we conclude that, of all commercial CD95L antibodies tested in this study, G247-4 is the only one with satisfying specificity and sensitivity in immunhistochemistry on frozen and paraffin sections.

\section{Materials and Methods}

\section{Cells and tissues}

CV-1/EBNA cells were co-transfected essentially as described. ${ }^{14}$ Here, we transfected the cells on cover slides and used a mix of three different plasmids in each transfection: (i) either one of the following three plasmids: (a) pCDNA3 (Invitrogen, San Diego, CA, USA), or pCDNA3 that encoded either full length human CD95L (b) or full length human TRAIL (c); (ii) a plasmid that encodes the anti-apoptotic Baculovirus protein $\mathrm{p} 35$, and (iii) pSV3-Neo that encodes the large T antigen necessary for the replication of pCDNA3. Cells were fixed in acetone $48 \mathrm{~h}$ after transfection and then subjected to immunocytochemical stainings.

Tissue samples from human tonsils were surgically removed and snap frozen in liquid nitrogen or fixed in $4 \%$ neutral buffered formalin within $30 \mathrm{~min}$ from surgery. Frozen sections of about $5 \mu \mathrm{m}$ were prepared, fixed in acetone for $10 \mathrm{~min}$, and stored at $-20^{\circ} \mathrm{C}$ until use. 
Formalin fixed tissues were dehydrated in ethanol and xylene and finally embedded in paraffin as usual. Paraffin-embedded tissue samples were cut into sections of about $3-4 \mu \mathrm{m}$ and processed as described below.

\section{RT-PCR for human CD95L and TRAIL}

CD95L and TRAIL expression of transfected cells was controled by performing RT-PCR. Cellular total RNA and DNA were extracted with TRIzol ${ }^{\mathrm{TM}}$ reagent (Life Technology, Gaithersburg, MD, USA) according to the manufacturer's instructions. RNA was treated with RNAse-free DNAse I (Boehringer-Mannheim, Mannheim, Germany) and reversely transcribed using a SuperScript ${ }^{\mathrm{TM}}$ Reverse Transcriptase (Life Technology) and random hexamer primers following the manufacturer's protocol. PCR was performed on CDNA, nontranscribed RNA, and DNA using the Taq PCR Core Kit (Quiagen, Hilden, Germany) and the following primer pairs, respectively: human CD95L: 5'-GAAGAAGAGAGGGAACCAC-3' and 5'-ATCTTCCCCTCCATCATC-3'; human TRAIL: 5'-TGACGAAGAGAGTATGAACAG-3' and $5^{\prime}$-TGGTCCCAGTTATGTGAG-3'. PCR products were resolved on a $2 \%$ agarose gel and visualized with ethidium bromide. The expected length of the amplicons were $474 \mathrm{bp}$ and $185 \mathrm{bp}$, respectively.

\section{Immunocyto/histochemistry}

Two polyclonal and 10 monoclonal CD95L antibodies, listed in Table 1 , were used in this study.

Immunocyto/histochemistry was carried out on slides with transfected cells, frozen and paraffin sections. While aceton fixed slides and frozen sections were directly incubated with anti-CD95L antibodies at appropriate concentrations, formalin fixed tissue was pretreated as follows. Prior to incubation with primary antibody, paraffin sections were dewaxed in xylene and rehydrated in ethanol/ water. Paraffin sections for stainings with monoclonal antibodies were then treated by microwave irradiation $(750 \mathrm{~W}, 20 \mathrm{~min})$ or cooked in a pressure cooker for $20 \mathrm{~min}$ in citrate buffer $(\mathrm{pH} 6.0)$, respectively, as described. ${ }^{11,15}$

Alternatively, sections were digested for $5 \mathrm{~min}$ by pronase $\mathrm{E}$ (Sigma, St. Louis, MO, USA; $1 \mathrm{mg} / \mathrm{ml}$ in PBS) or left untreated. In the case of G247-4 and $\mathrm{mAb} 33$, best staining results were yielded with microwave irradiation. With NOK-1, NOK-2, MIKE-1, and 4H9, 'antigen retrieval' by either method did not improve results compared to slides without pretreatment. Stainings with the polyclonal antibodies C-20 and $\mathrm{N}-20$ were preceded by incubating tissue sections with $5 \%$ normal goat serum as described by Bennett et al. ${ }^{16,17}$
Staining procedure was as follows: cells/sections were incubated with antibody in PBS for $60 \mathrm{~min}$ at room temperature. Different concentrations (given in Table 1) of each antibody were tested. Best signal-to-noise ratios were yielded using $20 \mu \mathrm{g} / \mathrm{ml}$ of monoclonal and $5 \mu \mathrm{g} / \mathrm{ml}$ of polyclonal antibodies, respectively. Binding sites of the primary antibody were visualized using appropriate biotinylated secondary antibodies (to rabbit, rat, mouse, and armenian hamster Ig, respectively) (Dako, Copenhagen, Denmark) and a streptavidin peroxidase system (Amersham, Buckinghamshire, UK). Threeaminoethylcarbazole (Sigma) was used as a substrate for the peroxidase which gave rise to a red precipitate at antibody binding sites. Cells/sections were then counterstained with Harris' hematoxylin and mounted with gelatin. Controls were done by omitting the primary antibody.

\section{In situ hybridization}

In situ hybridization was performed on transfected cells as well as on dewaxed and rehydrated paraffin sections as follows: CD95L mRNA was hybridized with the exon 4-specific digoxigenin-labeled human CD95L probe 1.APO- $1 \mathrm{~L}$ at $0.05 \mu \mathrm{M}$ as previously described. ${ }^{11,18}$ After incubation with a gold-labeled sheep anti-digoxigenin antibody (Aurion, Wageningen, Netherlands), bound gold particles were visualized for light microscopy using a silver enhancement kit (Aurion). Sections were then slightly counterstained with $2 \%$ pyronine $Y$ solution (Fluka, Buchs, Switzerland) and mounted with gelatin. Negative controls were carried out using (i) the unlabeled probe and (ii) the digoxigenin-labeled CD95L promoter-specific oligonucleotide, Prom2, and did not yield any signal above background levels (for further details $c f^{18}$ ).

\section{Acknowledgements}

The authors thank S Westenfelder, K Pruy, and $\mathrm{H}$ Stahl for excellent technical assistance. The study was supported by grants from the Deutsche Forschungsgemeinschaft (D.0687 STR 543/1-1; SFB518/A6). $\mathrm{H}$ Walczak was supported by a BioFuture grant from the Bundesministerium für Bildung und Forschung (BMBF).

Table 1

\begin{tabular}{|c|c|c|c|c|c|}
\hline Clone & Source & Isotype & Antigen & Supplier & $\begin{array}{c}\text { Concentrations } \\
(\mu \mathrm{g} / \mathrm{ml})\end{array}$ \\
\hline C-20 & rabbit & (polyclonal) & human & Santa Cruz & $0.2 ; 1 ; 5 ; 10$ \\
\hline $\mathrm{N}-20$ & rabbit & (polyclonal) & rat & Santa Cruz & $0.2 ; 1 ; 5 ; 10$ \\
\hline A11 & rat & $\lg \mathrm{M}$ & human & Alexis & $10 ; 20 ; 50$ \\
\hline $\mathrm{H} 11$ & rat & $\lg G 2 a$ & human & Alexis & $10 ; 20 ; 50$ \\
\hline MIKE-1 & rat & $\lg G$ & human & Alexis & $10 ; 20 ; 50$ \\
\hline MIKE-2 & rat & $\lg \mathrm{G} 2 \mathrm{a}$ & human & Alexis & $10 ; 20 ; 50$ \\
\hline $8 B 8$ & mouse & $\lg G 1$ & human & Calbiochem & $10 ; 20 ; 50$ \\
\hline $4 \mathrm{~A} 5$ & armenian hamster & $\lg G$ & human & Immunotech & $10 ; 20 ; 50$ \\
\hline $4 \mathrm{H} 9$ & armenian hamster & $\lg G$ & human & Immunotech & $10 ; 20 ; 50$ \\
\hline G247-4 & mouse & $\lg G 1$ & human & Pharmingen & $10 ; 20 ; 50$ \\
\hline NOK-1 & mouse & $\lg G 1$ & human & Pharmingen & $10 ; 20 ; 50$ \\
\hline NOK-2 & mouse & $\lg G 2 a$ & human & Pharmingen & $10 ; 20 ; 50$ \\
\hline 33 & mouse & IgG1 & human & Transduction Lab. & $10 ; 20 ; 50$ \\
\hline
\end{tabular}




\section{References}

1. Suda T, Takahashi T, Golstein P and Nagata S (1993) Molecular cloning and expression of the Fas ligand, a novel member of the Tumor Necrosis Factor family. Cell 75: $1169-1178$

2. Takahashi T, Tanaka M, Inazawa J, Abe T, Suda T and Nagata S (1994) Human Fas ligand: gene structure, chromosomal location and species specificity. Int. Immunol. 10: 1567-1574

3. van-Parijs L, Abbas -AK (1996) Role of Fas-mediated cell death in the regulation of immune responses. Curr. Opin. Immunol. 8: 355-361

4. Griffith TS and Ferguson TA (1997) The role of FasL-induced apoptosis in immune privilege. Immunol. Today 18: 240-244

5. O'Connell J, Bennett MW, O'Sullivan GC, Collins JK and Shanahan F (1999) The Fas counterattack: cancer as a site of immune privilege. Immunol. Today 20:4652

6. Stokes TA, Rymaszewski M, Arscott PL, Wang SH, BretzJD, Bartron Jand Baker Jr JR (1998) Constitutive expression of FasL in thyrocytes. Science 279: 2015

7. Fiedler P, Schaetzlien CE and Eibel H (1998) Constitutive expression of FasL in thyrocytes. Science 279: 2015

8. Smith D, Sieg Sand Kaplan D (1998) Aberrant detection of cell surface Fas ligand with anti-peptide antibodies. J. Immunol. 160: 4159-4160

9. Fiedler $\mathrm{P}$ and Eibel $\mathrm{H}$ (2000) Antibody $\mathrm{mAb33}$ from Transduction Laboratories detects human CD95L in ELISA but not in immunoblots. Cell Death Differ. 7: $126-128$

10. Baker Jr JR and Bretz JD (2000) Specificity questions concerning the clone 33 anti-fas ligand antibody. Cell Death Differ. 7: 8-9

11. Sträter J, Mariani SM, Walczak H, Rücker FG, Leithäuser F, Krammer PH and Möller P (1999) CD95 ligand (CD95L) in normal human lymphoid tissues: a subset of plasma cells are prominent producers of CD95L. Am. J. Pathol. 154: 193-201
12. Giordano C, Stassi G, DeMaria R, Toldaro M, Richiusa P, Papoff G, Ruberti G, Bagnasco M, Testi R and Galluzzo A (1997) Potential involvement of Fas and its ligand in the pathogenesis of Hashimoto's thyroiditis. Science 275: 960-963

13. Herr I, Posovsky C, Bohler T, Debatin K-M (2000) mAb 33 from Transduction Laboratories specifically binds to human CD95-L. Cell Death Differ. 7: 129-130

14. Walczak H, Degli-Esposti MA, Johnson RS, Smolak PJ, Waugh JY, Boiani N, Timour MS, Gerhart MJ, Schooley KA, Smith CA, Goodwin RG and Rauch CT (1997) TRAIL-R2: a novel apoptosis-mediating receptor for TRAIL. EMBO J. 16: $5386-5397$

15. Younes M, Schwartz MR, Ertan A, Finnie D and Younes A (2000) Fas ligand expression in esophageal carcinomas and their lymph node metastases. Cancer 88: $524-528$

16. Bennett MW, O'Connell J, O'Sullivan GC, Brady C, Roche D, Collins JK and Shanahan F (1998) The Fas counterattack in vivo: apoptotic depletion of tumorinfiltrating lymphocytes associated with Fas ligand expression by human esophageal carcinomas. J. Immunol. 160: 5669-5675

17. Bennett MW, O'Connell J, O'Sullivan GC, Roche D, Brady C, Kelly J, Collins JK and Shanahan $F$ (1999) Expression of Fas ligand by human gastric adenocarcinomas: a potential mechanism of immune escape in stomach cancer. Gut 44: 156-162

18. Möller P, Walczak H, Riedl S, Sträter J and Krammer PH (1996) Paneth cells express high levels of CD95 ligand transcripts. A unique property among gastrointestinal epithelia. Am. J. Pathol. 149: 9-13 